\title{
Zu überarbeiteten Standarddatenschutzklauseln und zur Data-Mining Entscheidung des BVerfG
}

\section{Stellungnahme zu überarbeiteten Standarddatenschutzklauseln}

Der Bundesbeauftragte für den Datenschutz und die Informationsfreiheit (BfDI), Professor Ulrich Kelber, ist sehr zufrieden mit dem Ergebnis des Europäischen Datenschutzausschusses (EDSA) zu Standarddatenschutzklauseln (SDK). Die europäischen Datenschutzaufsichtsbehörden und der Europäische Datenschutzbeauftragte (EDPS) hatten in der Sitzung vom 14. Januar gemeinsame Stellungnahmen zu den Entwürfen der Europäischen Kommission von SDK beschlossen.

Dazu sagte BfDI Ulrich Kelber am 15. Januar 2021: Es gab intensive Verhandlungen zu den Stellungnahmen, die ich mit meinen Kolleginnen und Kollegen verabschiedet habe. EDSA und EDPS kommen zu einem klaren Urteil. Unsere deutsche Position findet sich an vielen Stellen der Papiere wieder. Dieser gemeinsame Vorschlag würde Rechtssicherheit für den Datenaustausch mit Ländern außerhalb des Europäischen Wirtschaftsraumes bringen, ohne Einschränkungen beim Datenschutz zu machen.

Der BfDI hatte mit seinen Kolleginnen und Kollegen aus den Bundesländern die deutsche Position entwickelt. Der EDSA und der EDPS waren zum Jahreswechsel von der Europäischen Kommission gebeten worden, eine gemeinsame Stellungnahme zu zwei Entwürfen von SDK nach Art. 28 und Art. 46 der Datenschutz-Grundverordnung (DSGVO) zu erarbeiten. Die neuen SDK zu Art. 46 DSGVO sollen die bisherigen bei internationalen Datenübermittlungen ersetzen. Neuerungen gibt es beispielsweise bei Anpassungen an die Anforderungen der DSGVO und die Schrems II Rechtsprechung des Europäischen Gerichtshofes. Die SDK zu Artikel 28 DSGVO sollen für die Vertragsgestaltung zwischen Verantwortlichen und Auftragsverarbeitern erstmals einen europaweit verwendbaren Standard setzen, der den Unternehmen und Behörden die Umsetzung der entsprechenden Vorgaben der DSGVO deutlich erleichtert. Der EDSA wird den gemeinsamen Vorschlag in Kürze auf seiner Homepage veröffentlichen: $h t t p s: / / e d p b . e u r o p a . e u /$

\section{BfDI begrüßt Beschluss zu Data Mining}

Der Bundesbeauftragte für den Datenschutz und die Informationsfreiheit (BfDI), Professor Ulrich Kelber, sieht sich durch die Entscheidung des Bundesverfassungsgerichts vom 11. November 2920 zum so genannten „Data Mining“ in seiner Rechtsauf- fassung bestätigt. Die Richter entschieden, dass die erweiterte Datennutzung nach dem Antiterrordateigesetz teilweise verfassungswidrig sei.

Dazu sagte der BfDI: Die heutige Entscheidung stärkt den Datenschutz. Das Bundesverfassungsgericht hat sich erstmals zu Data Mining-Anwendungen innerhalb von Datenbanken der Sicherheitsbehörden geäußert. Es hat meine seit langem vertretene Auffassung bestätigt: Die Analyse von personenbezogenen Daten mit entsprechenden Techniken stellt einen Eingriff in das Recht auf informationelle Selbstbestimmung dar. Solche Techniken bedürfen einer klaren Rechtsgrundlage mit eigenständigen Eingriffsschwellen. Der Gesetzgeber ist gehalten, die klaren Vorgaben des Verfassungsgerichts endlich vollumfänglich umzusetzen. Die Antiterrordatei könnte ganz entfallen, da die Sicherheitsbehörden überwiegend schon besser geeignete Instrumente zur Kooperation nutzen.

Die heutige Entscheidung zur so genannten erweiterten Datennutzung gemeinsamer Dateien durch Polizeibehörden und Nachrichtendienste reiht sich in die verfassungsgerichtliche Rechtsprechung der letzten Jahre ein. Dabei hat das Bundesverfassungsgericht immer wieder betont, dass Eingriffe in die Datenschutzrechte der betroffenen Personen an ihrer Intensität zu messen sind. Intensive Eingriffe - wie etwa die erweiterte Datenanalyse - bedürfen einer hinreichend eindeutigen und verhältnismäßigen Rechtsgrundlage. Diese muss klare Eingriffsschwellen für die handelnden Behörden vorsehen. Umfangreiche Datenauswertungen ohne gewichtigen Anlass sind unzulässig. Das bedeutet für die Sicherheitsbehörden, dass sie neue Analysesysteme mit entsprechenden Funktionalitäten nicht ohne besondere Rechtsgrundlage einführen dürfen.

Hintergrund des Beschlusses des Ersten Senats war eine Verfassungsbeschwerde gegen eine Vorschrift des Antiterrordateigesetzes. Diese Vorschrift regelt die projektbezogene Nutzung von Daten aus der Antiterrordatei. Eingeführt wurde die Vorschrift bei der Novellierung des Antiterrordateigesetzes im Jahr 2014 mit der Begründung, dass die Notwendigkeit bestehe, auch komplexe Abfragen über den Datenbestand der Antiterrordatei durchzuführen. Aufgrund fehlender technischer Parameter war die Regelung in der Praxis bis heute nicht umgesetzt worden.

Prof. Ulrich Kelber, Der Bundesbeauftrage für den Datenschutz und die Informationsfreiheit 